\title{
Uso de Extractos de Pseudomonas sp (PB11) para el Control de la Mancha Bacteriana en tomate (Solanum lycopersicum)
}

\author{
Guadalupe Oyoque $^{(1)}$, Hortencia G. Mena ${ }^{(1)}$ Víctor Olalde $^{(2)}$, María V. Angoa ${ }^{(1) *}$ \\ (1) Centro Interdisciplinario de Investigación para el Desarrollo Integral Regional, IPN Michoacán, \\ Justo Sierra No. 28, CP 59519, Jiquilpan, Michoacán, México (e mail: goyoque@hotmail.com; \\ tenchisgmv@hotmail.com, valeangoa@hotmail.com) \\ (2)Centro de Investigación y de Estudios Avanzados del IPN Irapuato, México. Carretera Irapuato- \\ León, KM 9.6 libramiento Norte, CP 36000, Irapuato, Gto. México (e-mail: volalde@ipn.mx) \\ * Autor a quien debe ser dirigida la correspondencia
}

Recibido Ene. 18, 2011; Aceptado Mar. 01, 2011; Versión Final recibida Mar. 08, 2011

\section{Resumen}

Se ha usado extracto libre de células de la cepa patógena Pseudomonas sp (PB11) causante de la mancha bacteriana en tomate para control de la misma cepa en plantas de tomate (Solanum lycopersicum) en condiciones de invernadero. El extracto libre de células de PB11 fue aplicado a plantas de un mes de edad. Después de cuatro días, las plantas fueron inoculadas con una suspensión de Pseudomonas sp PB11. La severidad de la infección fue determinada por el número de lesiones necróticas presentes en cada planta y expresada como porcentaje de daño total. El extracto permitió la disminución de la infección en más del $60 \%(p<0.05)$. La protección se observó a partir del día cinco y se mantuvo durante los 30 días siguientes. Lo anterior muestra el potencial del extracto de PB11 para inducir una respuesta de defensa en plantas de tomate a Pseudomonas sp (PB11), en condiciones de invernadero.

Palabras clave: mancha bacteriana, tomate, extractos de cepas, Solanum lycopersicum, Pseudomonas $s p$

\section{Use of Pseudomonas sp (PB11) Extracts for Control of Bacterial Speck in Tomatoes (Solanum lycopersicum)}

\begin{abstract}
Cell free extract from a pathogenic strain of Pseudomonas sp (PB11) able to cause bacterial speck was prompted for protection against that disease in tomato plants (Solana lycopersicum) under greenhouse conditions. This extract was applied to one month old tomato plants. After four days, plants were inoculated with Pseudomonas sp PB11. Infection severity was determined by counting the number of necrotic lesions present in each plant and it were expressed as damage percentage. The extract led to a significantly infection reduction of more than $60 \%(p<0.05)$. The protection effect was observed on the fifth day after inoculation and it was maintained for the next 30 days. This study shows the potential of PB11 cell free extract to induce a defence response in tomato plants to Pseudomonas sp (PB11) under greenhouse conditions.
\end{abstract}

Keywords: bacterial speck, tomato, strain extracts, Solanum lycopersicum, Pseudomonas sp 


\section{INTRODUCCIÓN}

Las plantas son susceptibles a un gran número de patógenos, incluyendo hongos, bacterias, virus y nemátodos (Dmitriev, 2003). Para su control, se han usado una gran variedad de compuestos químicos que, al aplicarse de manera irracional, han causado daños al ambiente. Lo anterior ha motivado el interés por desarrollar métodos ambientalmente seguros y efectivos para manejar y controlar plagas y enfermedades vegetales, ya que el objetivo de la agricultura es lograr el equilibrio entre entre una producción competitiva y el respeto al medio ambiente (Simpaio, 2009). En este sentido, se ha explorado la posibilidad de usar productos que permitan inducir las defensas en las plantas como una estrategia segura para protegerlas (Hossain et al., 2008). Por otro lado, el uso de microorganismos supresores de fitopatógenos ha incrementado en los cultivos agrícolas. En este último caso, se ha reportado que ciertos microorganismos de los géneros Bacillus, Pseudomonas y Trichoderma pueden promover el desarrollo de las plantas al tiempo que ayudan a controlar enfermedades (Nakkeeran et al., 2005; Saravanakumar et al., 2007; van Loon, 2007).

Entre los mecanismos que dichos organismos emplean para proteger a las plantas de patógenos se encuentra el antagonismo por competencia, depredación o antibiosis. Pseudomonas fluorescens por ejemplo, provee control por su alta afinidad hacia los quelatos del ión hierro (sideróforos), lo cual limita las condiciones de crecimiento al patógeno; otro mecanismo de protección se lleva a cabo por la producción de metabolitos inhibidores del crecimiento tales como la fenazina -1-carboxamida producida por Pseudomonas chlororaphis y la pioquelina de Pseudomonas aeruginosa. Finalmente otro mecanismo es a través de la inducción de resistencia sistémica en plantas (Thomas et al., 2002).

La inducción de resistencia se manifiesta como una reducción de la incidencia o severidad de la infección de las plantas por un patógeno. Tal reducción en la susceptibilidad a una enfermedad puede ser local o sistémica, resultado del desarrollo de ciertas condiciones ambientales y dependiente de múltiples factores (van Loon, 2007). El potencial de las plantas para responder, radica en la producción de una señal liberada de las hojas infectadas y traslocada a otras partes de la planta donde se induce la respuesta de defensa. Esta forma de inducción de resistencia se conoce como Resistencia Sistémica Adquirida (RSA). Esta enfatiza el poder de la planta para adquirir un estado de resistencia general después de una infección inicial. De igual manera, bacterias no patógenas también son capaces de inducir una respuesta de resistencia en hojas (Pieterse and Van Loon, 1999) pero este tipo se denomina Resistencia Sistémica Inducida (RSI) (Métraux, 2001).

Dado lo anterior, las plantas son capaces de reconocer numerosos compuestos encontrados en estructuras de la superficie microbiana o metabolitos que producen durante el cultivo y que inducen una respuesta de defensa en la misma. Estos inductores pueden ser glicoproteínas, péptidos, lípidos y fragmentos de oligosacáridos de la pared celular, los cuales se encuentran ocasionalmente en extractos crudos y filtrados (Ben-Sholon et al., 2002; Dong et al., 2003; He et al., 2002). También se sabe que algunas bacterias patógenas pueden liberar moléculas activadoras de defensa en las plantas o pueden ser extraídas de tejido infectado a través del lavado de los espacios intracelulares, donde han colonizando los microorganismos (Chen et al., 2006; Riveros y Lepoivre, 1998; Waldmüller et al., 1992). En el caso de bacterias fitopatógenas como Xanthomonas campestris pv. vesicatoria se ha reportado su capacidad para secretar proteínas dependientes de genes Hrp (reacción hipersensible y patogenicidad) denominadas harpinas que participan en la inducción de resistencia (Alfano and Collmer, 1997).

Otro grupo de Pseudomonas fitopatógenas: $P$. putida, $P$. syringae, $P$. aeruginosa y $P$. corrugata, también son capaces de brindar protección a las plantas contra otros patógenos e incluso contra ellas mismas (Custers, 2007; Gray y Goodman, 2004). El hecho de que existan especies bacterianas patógenas capaces de brindar protección a plantas contra el ataque de ellas mismas resulta interesante, así como también el determinar si el uso de extractos libres de células del patógeno puede desencadenar el mismo efecto sobre la inducción de resistencia. Por lo antes mencionado, el objetivo de este trabajo fue estudiar el efecto del extracto libre de células obtenido de la cepa patógena de Pseudomonas sp (PB11) en el control de la enfermedad conocida como "mancha bacteriana" en tomate causada por la misma cepa.

\section{METODOLOGÍA}

La cepa de Pseudomonas sp utilizada en este estudio se obtuvo de hojas de plantas de tomate verde (Physalis ixocarpa Brot.) que presentaban lesiones características de la enfermedad denominada "mancha bacteriana" provocada por Pseudomonas sp., las cuales fueron recolectadas en 3 localidades de los estados de Jalisco y Michoacán, México. 


\section{Aislamiento e identificación de Pseudomonas sp PB11}

Los trozos de hojas aseptizadas fueron colocados en placas con medio de extracto de levadura, dextrosa y carbonato de calcio (YDC), se incubaron a $25^{\circ} \mathrm{C}$ durante $48 \mathrm{~h}$ (Agrios, 2005). La cepa se identificó mediante la extracción de DNA cromosómico de cada cepa (Harwood and Cutting, 1990). Se utilizaron primers para amplificar la región 16S rDNA (Weisburg y col., 1991). Las condiciones utilizadas fueron las siguientes: 2 minutos $94^{\circ} \mathrm{C}, 30$ segundos $94^{\circ} \mathrm{C}, 45$ segundos $55^{\circ} \mathrm{C}, 72^{\circ} \mathrm{C}$ por 2 minutos y 30 repeticiones desde el paso 2 para finalizar con 5 minutos a $72^{\circ}$ C. Se realizó una mezcla de reacción de PCR $(20 \mu \mathrm{l})$, con $1 \mu \mathrm{l}$ de DNA cromosómico, con una concentración aproximada de 50ng, y 20 pmolar de cada primer. El producto de amplificación fue de aproximadamente 1500 pares de bases. El termociclador que se utilizó fue Minicycler MJ Research. Los productos de amplificación de 16S fueron clonados en el vector PCR 2.1 TOPO y secuenciados.

\section{Prueba de patogenicidad}

Para confirmar su patogenicidad, PB11 se inoculó en plantas de tomate (Solanum Lycopersicum L.) variedad rojo fuego de 1 mes de crecimiento, las cuales fueron sembradas en macetas de $4 \mathrm{~kg}$ con una mezcla de peat moss-(Pro-mix PGX) y perlita (Multiperl) $(1: 1 \mathrm{vv})$ estéril. Las plantas fueron asperjadas hasta punto de gota con una suspensión $1 \times 10^{8} \mathrm{UFC} / \mathrm{mL}$ y colocadas en cuarto de crecimiento a $27 \pm 1^{\circ}$ C hasta la aparición de lesiones características del ataque de Pseudomonas sp. Por cada tratamiento se asignaron 3 repeticiones. Al cabo de 20 días las plantas fueron cosechadas, y sus hojas fueron desinfectadas superficialmente y fragmentos de ellas fueron colocados en placas petri con medio YDC para el reaislamiento del patógeno de acuerdo a la técnica de Agrios, (2005) modificada.

\section{Obtención del extracto libre de células}

El extracto libre de células se preparó a partir de una suspensión de PB11 con $1 \times 10^{8}$ UFC/ml cultivada en Infusión Papa Dextrosa (PDI) (previamente esterilizado a $121^{\circ} \mathrm{C}$ por $15 \mathrm{~min}$ ) por un periodo de $12 \mathrm{~h}$ a temperatura de $25^{\circ} \mathrm{C}$ con agitación constante de 200 vaivenes/min. La suspensión se centrifugó en una centrífuga Wheaton RMC-2 a $10000 \mathrm{rpm} / 10 \mathrm{~min}$ y el sobrenadante se filtró a través de membranas de nylon Millipore estériles de $0.22 \mu \mathrm{m}$ (Millipore Corporation, Bedford, MA) con ayuda de vacío (40 cm de Hg). Finalmente se esterilizó a $121^{\circ} \mathrm{C}$ por 15 min para eliminar cualquier célula remanente. De igual manera PDI fue filtrado y esterilizado en autoclave.

\section{Evaluación de inducción de resistencia}

Se desinfectaron semillas de tomate variedad rojo fuego con solución de $\mathrm{NaClO}$ al $3 \%$ durante 5 min con posterior enjuague de agua destilada estéril. Se sembraron en una mezcla estéril (1:1) de peat moss (Pro-mix PGX) y perlita (Multiperl) contenido en macetas de $4 \mathrm{~kg}$. Las plantas se colocaron en invernadero para su crecimiento dentro de bolsas de polietileno de $1 \times 1.20 \mathrm{~m}$ con ventanas laterales de malla antiáfido de $15 \times 15 \mathrm{~cm}$ y a temperatura de $22-35^{\circ} \mathrm{C}$ para protegerlas del ataque de alguna plaga. Las plantas de 1 mes de edad recibieron los siguientes tratamientos:

1.- Plantas asperjadas con Infusión Papa Dextrosa, PDI

2.- Plantas asperjadas con una suspensión de $1 \times 10^{8} \mathrm{UFC} / \mathrm{mL}$ de Pseudomonas sp PB11 (PB11)

3.- Plantas asperjadas hasta punto de gota con el extracto libre de células procedente de la cepa PB11. Después de 4 días las plantas fueron inoculadas con una suspensión $1 \times 10^{8} \mathrm{UFC} / \mathrm{mL}$ de la misma cepa.

Cada tratamiento constó de 5 plantas, cada planta representó una repetición. Para evitar un estrés nutricional, las plantas fueron fertilizadas cada 3 días con una concentración de $\mathrm{N}, \mathrm{P}$ y K de 200 , 80 y 400 ppm, usando como fuente $\mathrm{CaNO}_{3}, \mathrm{H}_{2} \mathrm{PO}_{4}^{-}$, y $\mathrm{KNO}_{3}$ respectivamente. El progreso de la infección en todos los tratamientos fue evaluado a 1, 5, 10, 15, 20, 25 y 30 días. La severidad del daño ocasionado por el patógeno se determinó contando el número de manchas necróticas características por la presencia del patógeno en todas las hojas de las plantas de tomate y se 
Uso de Extractos de Pseudomonas sp (PB11) para el Control de la Mancha Bacteriana Oyoque

expresó como porcentaje de daño en toda la planta. El experimento se realizó 3 veces para confirmar la reproducibilidad del mismo. El diseño experimental fue completamente al azar y los datos obtenidos de todos los tratamientos en los 3 experimentos realizados $(n=15)$ se analizaron estadísticamente mediante una prueba de varianza a un nivel de significancia del 5\%. La comparación de medias se hizo mediante la prueba Tukey con el programa SAS system for Windows V9.

\section{RESULTADOS Y DISCUSIÓN}

De la comparación del producto de amplificación del 16S rDNA, con las bases de datos (BLAST, $\mathrm{nr} / \mathrm{nt}$ ), se encontró que PB11 (1431 pb) coincidió con una Pseudomonas sp. 1NT 16S RNA ribosomal. Como se muestra en la fig. 1, la aplicación del extracto libre de células de PB11 permitió la inducción de mecanismos de defensa en las plantas de tomate 5 días después de haber sido retadas con PB11 lo cual llevó a una evidente y significativa reducción de la severidad de infección en comparación con las plantas que solo tenían el patógeno. Dicha disminución se hizo más evidente con el tiempo, alcanzando una disminución de la severidad superior al $60 \%$ en el día 30 del experimento (Fig. 1). Al parecer, la aplicación del extracto libre de células sensibilizó a las plantas en los primeros días del experimento, permitiendo el inicio de infección por el patógeno, pero este efecto no fue duradero. Estos resultados son similares a los reportados por Thomas et al., (2002) y Sharathchandra et al., (2004) con la aplicación de diversos compuestos procedentes de diferentes patógenos los cuales indujeron mecanismos de defensa en las plantas contra otros patógenos.

Hay que destacar que, en este trabajo la inducción de la resistencia se dio hacia el mismo patógeno del cual se obtuvo el extracto libre de células y no contra otros patógenos, como fue el caso de los trabajos antes mencionados. Otro aspecto a destacar es que el efecto de protección fue duradero, ya que se mantuvo durante los 30 días del experimento (Fig. 1). En este sentido se ha reportado que la inducción de resistencia en las plantas con agentes activadores de defensa requiere de intervalos de tiempo variables para su establecimiento, antes de ser confrontadas con los patógenos. Cavalcanti et al., (2006) reportaron la inducción de resistencia de plantas de tomate hacia Xanthomonas vesicatoria (Doidge) por aplicación del extracto de Solanum lycocarpum infectado con Crinipellis perniciosa. Cabe señalar que el tiempo requerido por dicho extracto para inducir la resistencia fue igual al requerido por el extracto libre de células de PB11. Un resultado similar en el mismo intervalo de tiempo fue reportado con el uso de otros agentes como el quitosan y metil S-acibenzolar para brindar protección a plantas de tomate contra Xanthomonas sp. En este caso, se logró el control hasta en un $49.3 \%$ y $31.4 \%$ respectivamente (Cavalcanti et al., 2006; Cavalcanti et al., 2007). De igual manera, la aplicación de un extracto libre de células procedente de la bacteria promotora del crecimiento vegetal Phoma sp. GS8-1, indujo la resistencia de plantas de Arabidopsis thaliana a Pseudomonas syringae pv. Tomato con una reducción del $49 \%$ de los síntomas (Hossain et al., 2008). Es importante destacar que los niveles de control de la enfermedad reportados en los trabajos antes mencionados fueron menores al obtenido en este trabajo que fue de mayor al $60 \%$.

Cabe señalar que existen trabajos como el de Schuhegger et al., (2006) en donde la aplicación de compuestos producidos por bacterias rizosféricas (ejemplo la $\mathrm{N}$-acil-L lactona) han sido capaces de inducir los mecanismos de defensa contra hongos como Alternaria alternata con exposición al extracto bacteriano de 3 días, e incluso menos tiempo gracias al uso de mutantes no patogénicas de Xanthomonas campestris pv. vesicatoria ( $h r p G, h r p F)$ y $P$. syringae pv. syringae, para protección contra C. michiganense pv. Michiganense (Ercolani, 1969; Moss et al., 2007). Las investigaciones sugieren que el intervalo en que puede desencadenarse la inducción de la resistencia puede oscilar entre 1-7 días (Baysal et al., 2003; Silva et al., 2003; Sharathchandra et al., 2004). Es importante destacar que, a diferencia de los trabajos antes mencionados donde se recurre a la aplicación directa de las bacterias ya sean patógenas o promotoras del crecimiento vegetal para el control de patógenos, distintos a los aplicados en su caso, en este trabajo se emplea el extracto libre de células de una bacteria patógena para el control de la misma. 
Otro aspecto importante de resaltar de este trabajo es el referente al tiempo de inducción para alcanzar un umbral verdadero de protección. En este sentido hay discrepancias entre autores pero dicho intervalo puede oscilar de unos días hasta seis semanas (Tally et al., 2000). En este trabajo la respuesta se mantuvo durante los 30 días de observación, lo cual implicaría una reducción de aplicaciones del producto y a futuro, un beneficio económico. Con respecto a la naturaleza de los compuestos responsables de la inducción de resistencia en el caso del extracto libre de células de la cepa PB11 aun no se conocen, pero debe hacerse hincapié en que se trata de activador (es) de defensa provenientes del patógeno que se desea controlar, no obstante no se descarta la posibilidad de que funcione también para la inducción de resistencia sistémica en la planta contra otros patógenos al desencadenar el mecanismos de resistencia adquirida (Camarena- Gutiérrez y de la Torre-Almaraz 2007). Sin embargo, hace falta profundizar en el estudio para probar esta tesis. De igual manera resulta interesante determinar a nivel molecular los posibles mecanismos por los cuales el extracto bacteriano libre de célula indujo la resistencia en las plantas a las cuales se aplicó, así como la naturaleza de los compuestos inductores.

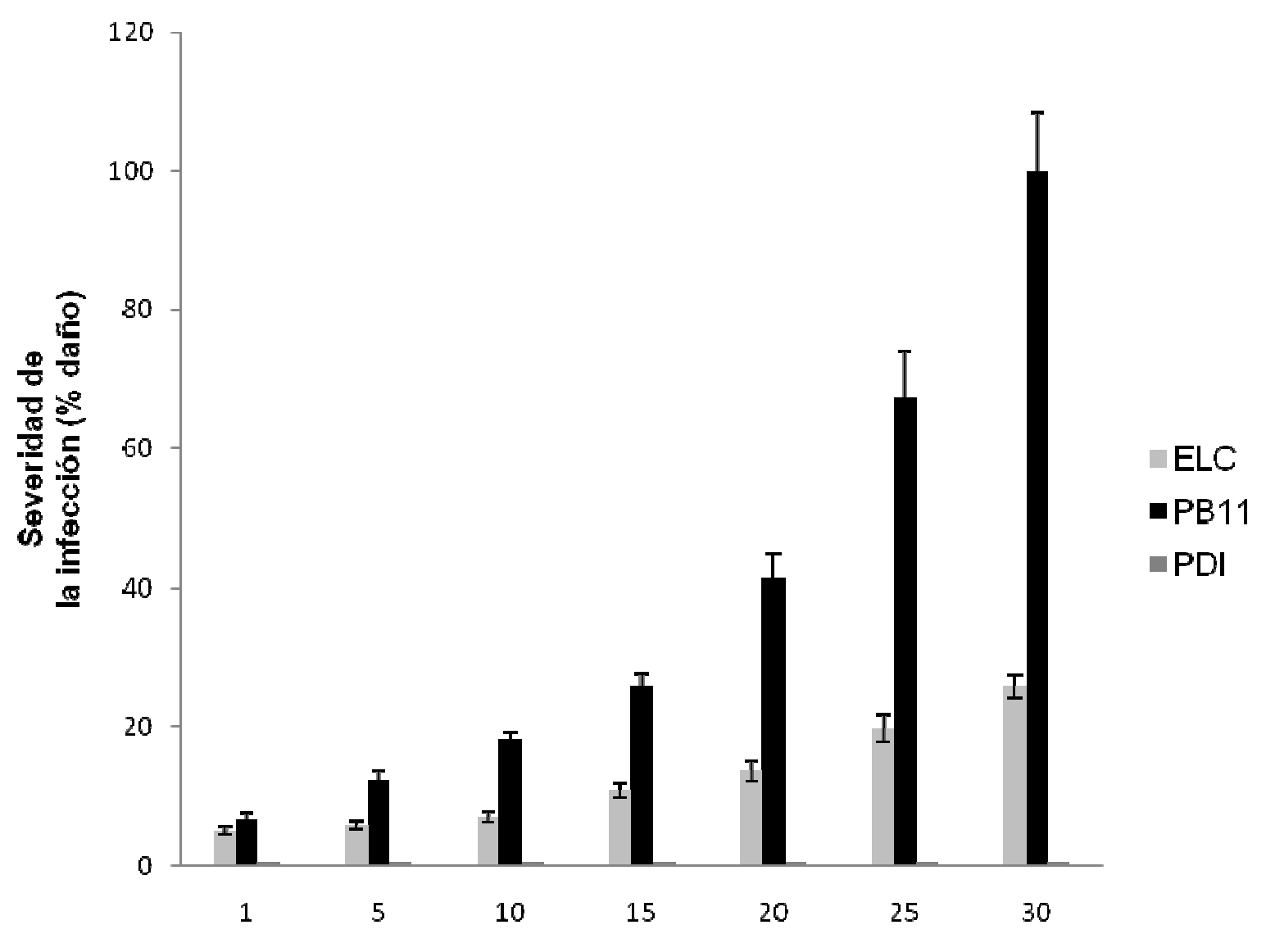

Días de muestreo

Fig. 1: Severidad de la infección (Porcentaje de daño) de plantas de tomate infectadas con Pseudomonas sp (PB11), asperjadas con el extracto libre de células proveniente de la cepa PB11 (ECL) y con el medio de cultivo (PDI) durante 30 días de duración del experimento. Los datos son presentados como la desviación estándar $\pm(p<0.05 ; n=15)$.

\section{CONCLUSIONES}

La aplicación del extracto libre de células de la cepa patógena Pseudomonas sp. PB11 permitió disminuir el daño que dicha cepa ocasiona en plantas de tomate. La protección comenzó a partir del día 5 del experimento y se mantuvo durante los 30 días evaluados. Lo anterior muestra el potencial del extracto libre de células de PB11 para inducir una respuesta de defensa en plantas de tomate a la mancha bacteriana, no obstante hace falta realizar pruebas en campo y explorar la inducción de la resistencia en otros cultivos. 


\section{AGRADECIMIENTOS}

Agradecemos al Instituto Politécnico Nacional y al Consejo Nacional de Ciencia y Tecnología de México por el financiamiento otorgado para la realización de este trabajo. Agradecemos también a la Dra. Mariana Angoa por los valiosos comentarios y sugerencias para mejorar el documento.

\section{REFERENCIAS}

Agrios, G.N., Plant pathology. 635. Harcourt/Academic Press, USA (2005).

Alfano, J.R. y A. Collmer, The type III (Hrp) secretion pathway of plant pathogenic bacteria: trafficking harpins, Avr proteins and death, J. of Bacteriology 179, 5655-5662 (1997).

Baysal, Ö., E.M. Soylu y S. Soylu, Induction of defence-related enzymes and resistance by the plant activator acibenzolar-S-methyl in tomato seedlings against bacterial canker caused by Clavibacter michiganensis ssp. Michiganensis, Plant pathology 52, 747-753 (2003).

Baysal, Ö. y otros cuatro autores, Enhanced siystemic resistance to bacterial speck disease caused by Pseudomonas syringae pv. Tomato by DL- $\beta$-aminobutyric acid under salt stress, Physiol. Plantarum 129, 493-506 (2007).

Ben-Sholon, N., C. Aki, R. Ardi y R. Pinto, Elicitation effects of chitin oligomers and chitosan sprayed on the leaves of cucumber (Cucumis sativus) and bean (Phaseolus vulgaris) plants, Israel J. Plant Sci. 50, 199-206 (2002).

Camarena- Gutiérrez, G. y R. de la Torre-Almaraz, Resistencia sistémica adquirida en plantas: estado actual, Revista Chapingo 13,157-162 (2007).

Cavalcanti, F.R. y otros cuatro autores, An aqueous suspensión of Crinipellis perniciosa mycelium activates tomato defence responses against Xanthomonas vesicatoria, Crop Protection 26, 729738 (2007).

Cavalcanti, F.R. y otros cuatro autores, Induced defense responses and protective effects on tomato against Xanthomonas vesicatoria by an aqueous extract from Solanum lycocarpum infected with Crinipellis perniciosa, Biological Control 39, 408-417 (2006).

Custers, J.H.H.V., General introduction: engineering disease resistance in plants. In: Engineering disease resistance in plants, By J.H.H.V. Custers, pp145-156, Syngenta, Netherland (2007).

Chen, S. y otros cuatro atores, Dry mycelium of Penicillium chrysogenum induces expression of pathogenesis-related protein genes and resistance against wilt diseases in Bt transgenic cotton, Biol. Control 39,460-464 (2006).

Dmitriev, A.P., Signal Molecules for Plant Defense Responses to Biotic Stress, Russian J.Plant Physiol. 50(3), 417-425 2003. Traducido de Fiziologiya Rastenii 50(3), 465-474 (2003).

Dong, $\mathrm{H}$., y otros tres autores, Differential expression of induced resistance by an aqueous extract of killed Penicillium chrysogenum against Verticillium wilt of cotton, Crop Protection 22, 129-134 (2003).

Ercolani, G.L., Studies on bacterial canker of tomato: A progress report, Annu. Phytopathology 1, 255-263 (1969).

Gray, E. y R.M.Goodman, Systemic Acquired Resistance and Induced Systemic Resistance in conventional Agriculture, Crop Sci. 44, 1920-1933 (2004).

Harwood, C.R y S. Cutting, Genetic Analysis. In: Molecular Biological Methods for Bacillus, C.R Harwood y S. Cutting, pp 27-61. A Wiley-Interscience Publication, England (1990). 
He, C., Y.T. Hsiang y D.J. Wolyn, Induction of systemic disease resistance and pathogen defence responses in Asparagus officinalis with nonpathogenic strains of Fusarium oxysporum, Plant Pathol. 51, 225-230 (2002).

Hossain, M. y otros tres autores, Differential inducible defense mechanisms against bacterial speck pathogen in Arabidopsis thaliana b plant-growth-promoting-fungus Penicillium sp. GP16-2 and its cell free filtrate, Plant Soil 304, 227-239 (2008). DOI 10.1007/s11104-008-9542-3

Métraux, J.P., Systemic acquired resistance and salicylic acid: current state of knowledge, Eur. J. Plant Pathol. 107, 13-18 (2001).

Moss, W.P. y otros seis autores, Biological control of bacterial spot of tomato using hrp mutants of Xanthomonas campestris pv. Vesicatoria, Biol. Control 41,199-206 (2007).

Nakkeeran, S., P. Renukadevi y T. Marimuthu, Antagonistic potentiality of Trichoderma viride and assessment of its efficacy for the management of cotton root, Arch. Phytopathol. Plant Protection 38, 209-225 (2005).

Pieterse, C.M.J. y L.C. van Loon, Salicylic acid-independent plant defence pathways, Trends in Plant Sci. 4, 52-58 (1999).

Riveros, A.S. y P.H. Lepoivre, Alternativas bioquímicas para el control indirecto de Sigatoka en Musáceas. Reunión ACORBAT, 436-447, Guayaquil, Ecuador 8 (1998).

Saravanakumar, D. y otros tres autores, PGPR-induced defense responses in the tea plant against blister blight disease, Crop Protection 26,556-565 (2007).

Schuhegger, R. y otros once autores, Induction of systemic resistance in tomato by N-acyl-Lhomocesine lactone-producing rhizosphere bacterial, Plant Cell Environ. 29, 909-918 (2006).

Sharathchandra, R.G. y otros 4 autores, A Chitosan formulation Elexa induces downy mildew disease resistance and growth promotion in pearl millet, Crop Protection 23, 881-888 (2004).

Silva, L.H.C.P. y otros cuatro autores, Indução de resistência contra Xanthomoas vesicatoria em tomateiro por acibenzolar-S-metil. Summ, Phytopathol. 29, 177-181 (2003).

Simpaio, E.P. Estudio de las prácticas culturales en sus relaciones con agricultura, suelo y ambiente. Inf. Tecnol. 20 (3), 113-123 (2009)

Tally, A. y otros cuatro autores, Commercial development of elicitors of induced resistance to pathogens. In plant defenses against pathogens and herbivores. Biochemistry, Ecology and Agriculture. by A.A. Agrawal, S. Tuzun y E. Bent pp 357-369 APS Press, St Paul Miennnesota, USA (2000).

Thomas, F.C. y otros tres autores, Phenazine and their role in biocontrol by Pseudomonas bacteria, Rev. New Phytol. 157, 503-523 (2002).

Van Loon, L.C. Plant responses to plant growth-promoting rhizobacteria, Eur. J.I Plant Pathol. 119, 243-254 (2007). DOI 10.1007/s10658-007-9165-1

Waldmüller, T.E. y otros tres autores, Release of highly elicitor-active glucans by germinating zoospores of Phytophthora megasperma f.sp. glycinea, Planta 188, 498-505 (1992).

Weisburg, W.G. y otros tres autores. 16S ribosomal DNA amplification for phylogenetic study. J. Bacteriol. 173, 697-703 (1991). 
